\title{
Raíz secundaria embrional asociada a la germinación de Garcinia intermedia (Clusiaceae) y su posible papel en la supervivencia de las plántulas
}

\author{
José Fco. Di Stefano, Walter A. Marín \& Marco A. Díaz \\ Escuela de Biología, Universidad de Costa Rica; jdistefa@cariari.ucr.ac.cr; wmarin@cariari.ucr.ac.cr; \\ marcodiaz@walla.com
}

Recibido 13-IX-2004. ～Corregido 02-VI-2005. Aceptado 22-III-2006.

\begin{abstract}
Secondary embryonary root associated with seed germination in Garcinia intermedia (Clusiaceae) and its possible role in seedling survival. Germination tests on Garcinia intermedia (Clusiaceae) seeds showed the growth of two types of roots: additionally to the primary root, a secondary root crosses the seed lengthwise. To determine its possible role on the survival and growth of this species, 90 seedlings at least six months old (collected in Central Costa Rica) were planted in plastic bags with organic soil, and placed in a greenhouse. The seedlings were treated as follows: treatments in which the primary or secondary root was cut off, and a control group with both roots intact (30 replicates each). After three months 10 seedlings/month/treatment were extracted to measure their height, basal diameter, root length (main and secondary root), and biomass of the stem, roots and seed (without its coat). Control seedlings had the highest growth, followed by those without secondary roots. Nonetheless, more than $90 \%$ of the seedlings whose primary roots were cut off, survived after five months of the excision treatment, in part due to the capacity of this species to regenerate its radical system through the seed reserves, sprouting of a primary-like root, and/or the growth stimulus of the secondary root ( $60 \%$ of the total: $20 \%$ with sprouts from the primary root stump, $13.3 \%$ with a growth stimulus of the secondary root, and $26.7 \%$ with both conditions). The length of the sprouted roots was significantly different only on those plants that were extracted during the first two monthly measurements, when compared with the control ( $\left.F_{6}=18.6, F_{7}=16.0, p<0.01\right)$. Rev. Biol. Trop. 54(3): 927-934. Epub 2006 Sept. 29.
\end{abstract}

Key words: primary root, secondary root, root sprouting, hypogeous germination, Premontane humid tropical forest, Costa Rica.

En general, las fases más críticas del ciclo de vida de las plantas corresponden a las etapas de germinación y establecimiento de la plántula. Un número importante de semillas no completan su ciclo porque son depredadas o no encuentran el sitio adecuado para germinar, y las que germinan pueden sufrir una elevada mortalidad por variaciones en el clima, herbivoría, daños mecánicos o ataques por patógenos (Fournier y Salas 1967, Augspurger 1984, Dirzo 1987, Clark y Clark 1989, Farji-Brener et al. 2005, García-Robledo 2005).

Garcinia intermedia (Clusiaceae) mantiene poblaciones de plántulas muy abundantes en algunos sectores de la Zona Protectora "El Rodeo” (Di Stefano y Marín, obs. personal) por lo que se decidió estudiar algunos aspectos de su autoecología tales como su susceptibilidad a daños mecánicos, ataques por hongos y resistencia al estrés hídrico.

Al realizar pruebas preliminares de germinación en esta especie, se observó que las semillas desarrollan, aparte de su raíz primaria, otra raíz que atraviesa la semilla hasta salir por el extremo distal de la misma. A esta raíz se le consideró como una secundaria embrional (rs). En la literatura consultada, no se encontró ninguna referencia a este fenómeno, aunque aparentemente si ocurre en otras especies de la familia (E. Flores, com. pers.).

La presencia de este tipo de raíz plantea la interrogante sobre el papel ecológico que esta 
cumple en las fases iniciales del desarrollo de la plántula, razón por la cual se planteó como objetivo de la presente investigación, el determinar la influencia de esta sobre la supervivencia y crecimiento de la plántula cuando la raíz primaria es dañada.

\section{MATERIALES Y MÉTODOS}

A principios de mayo del 2003 se colectaron cerca de 400 semillas de G. intermedia (de frutos con una o dos semillas) a lo largo del sendero principal cercano al mirador (954'40.4'” N; 84¹6'46.4'” W, aproximadamente a $1000 \mathrm{msnm}$ ) en la Zona Protectora "El Rodeo”, en el cantón de Mora. Esta reserva se clasifica como un bosque Premontano húmedo (sensu Holdridge), y presenta una temperatura y precipitación total anual promedio de $23.7^{\circ} \mathrm{C}$ y $2150 \mathrm{~mm}$ (principalmente entre mayo y noviembre), respectivamente (datos suministrados por el Dr. Luis A Fournier de la Finca San Luis en Ciudad Colón, a 750 msnm; Cascante y Estrada 2001).

G. intermedia es un árbol de 5 a $12 \mathrm{~m}$ de altura distribuido desde México hasta Panamá en bosques de húmedos a muy húmedos de 0 a unos $1200 \mathrm{msnm}$, especialmente en la vertiente del Pacífico. Florea de enero a marzo y de julio a octubre (Zamora et al. 2004). Presenta un látex amarillo anaranjado muy abundante.

Las semillas $(1.54 \pm 0.49 \mathrm{~g}$ peso fresco, largo $2.23 \pm 0.27 \mathrm{~cm}$, ancho $1.27 \pm 0.17 \mathrm{~cm}$ ) fueron sembradas en bandejas con arena de río ubicadas en un invernadero de la Escuela de Biología de la Universidad de Costa Rica, Montes de Oca. Los epicótilos empezaron a emerger luego de mes y medio. A los tres meses, un promedio del $84 \pm 9.7 \%$ de las semillas produjo epicótilos visibles.

De ellas, se extrajeron 90 plántulas (las que poseían las mejores características). En promedio presentaban $8.38 \pm 1.86 \mathrm{~cm}$ de altura, $0.21 \pm 0.04 \mathrm{~cm}$ de diámetro basal, $6.15 \pm 0.41 \mathrm{~cm}$ de longitud raíz primaria, y $1.89 \pm 0.53 \mathrm{~cm}$ de longitud de la raíz secundaria embrional medida a partir del punto de salida de la semilla (Fig.
1). Éstas se plantaron en bolsas plásticas negras $(12.7$ x $20.3 \mathrm{~cm})$ con $3 \mathrm{~kg}$ de suelo orgánico cada una, y se colocaron en el invernadero. Se regaron al menos dos veces por semana, y se les arrancó las hierbas. Además, se controló el ataque de insectos, particularmente áfidos.

A las plántulas (tres meses de germinadas) se les aplicó uno de los siguientes tratamientos (cada una con 30 repeticiones):

Se les cortó la raíz primaria lo más cercano posible a la semilla (srp).

A. Se les cortó la raíz secundaria en el punto de salida de la semilla (srs).

B. Control (no se cortó ninguna de las raíces, ct).

Tres meses después de aplicados los tratamientos se extrajeron al azar 10 plántulas/mes/ tratamiento a las cuales se les midió la altura (ht), el diámetro basal (db), y la longitud de la raíz secundaria embrional (rs) y primaria (rp). Además, se les midió la longitud y ancho de la semilla, y la biomasa de todas las partes (vástago, raíces y semilla) luego de secarlas en una estufa a aproximadamente $60{ }^{\circ} \mathrm{C}$, hasta peso constante.

A los datos se le aplicó un ANDEVA de una vía si cumplían con el supuesto de normalidad (F). De lo contrario, se aplicó la prueba de Kruskal-Wallis $(\mathrm{H})$. Se usó el programa XLSTAT® 7.1 .

\section{RESULTADOS}

En este estudio se determinó que la germinación de G. intermedia es hipógea. Asimismo, se observó el desarrollo de dos tipos de raíces: la primaria y una secundaria la cual atravesaba la semilla hasta el exterior (Fig. 1).

La supervivencia de las plántulas a las que se les cortó la raíz primaria fue muy alta (superior al 90\% luego de cinco meses de haberse aplicado los tratamientos) a pesar del fuerte daño a las que se les sometió. Sin embargo, el crecimiento si se vio afectado (Fig. 2) en 


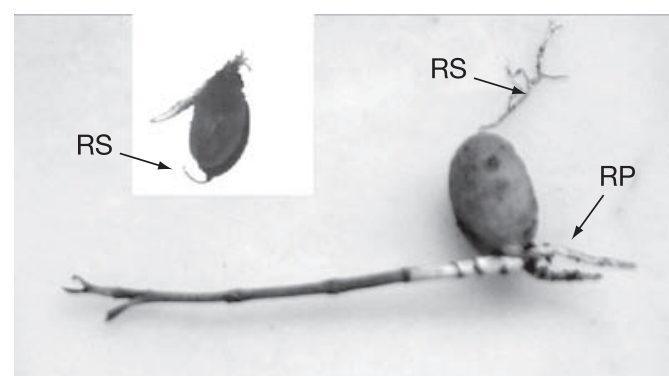

Fig. 1. Garcinia intermedia con al menos seis meses de germinada: raíz secundaria (RS) embrional y rebrotes de la raíz principal (RP). La figura inserta muestra una semilla recién germinada antes de aplicar el tratamiento.

Fig. 1. Garcinia intermedia six months after germination with the embrionary secondary root (RS) and sprouts of the primary root (RP). The insert shows a recently germinated seed before treatments were applied.
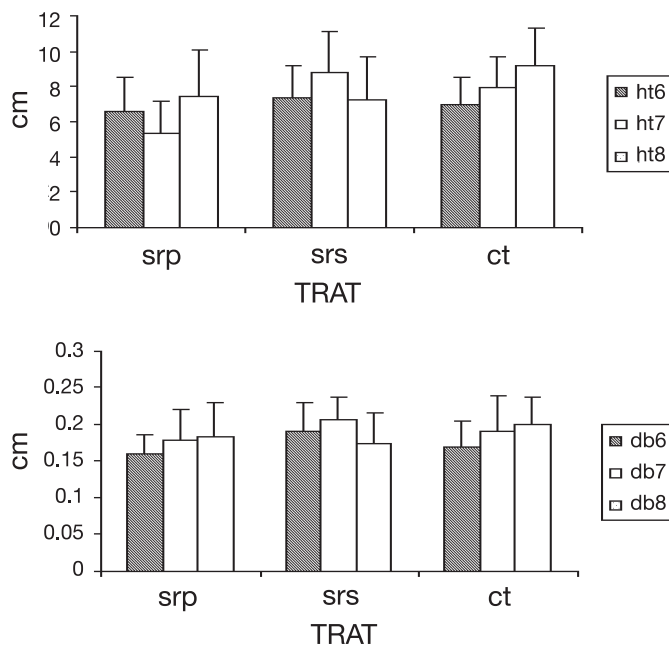

Fig. 2. Altura (ht) y diámetro basal (db) promedio (+ desviación estándar) de Garcinia intermedia en tres periodos de muestreo (6, 7 y 8 meses de germinado) según los tratamientos (TRAT: $\operatorname{srp}=$ sin raíz principal; srs= sin raíz secundaria; $\mathrm{ct}=$ control).

Fig. 2. Average (+ standard deviation) height (ht) and basal diameter (db) of Garcinia intermedia on three sampling periods (6, 7 and 8 months after germination) according to treatments (TRAT: $\mathrm{srp}=$ without the primary root; srs= without the secondary root; $\mathrm{ct}=$ control).

especial de los 30 a 60 días después del corte. Este fue significativamente diferente para la altura a los siete meses de edad, y para el diámetro basal a los seis meses $\left(\mathrm{F}_{\mathrm{ht7}}=8.461, \mathrm{p}_{\mathrm{ht} 7}\right.$ $\left.=0.001 ; \mathrm{F}_{\mathrm{db} 6}=3.531, \mathrm{p}_{\mathrm{db} 6}=0.043\right)$.
Las plántulas srp mostraron una buena recuperación a este trauma en parte gracias a que un $60 \%$ de ellas recobraron el sistema radical a través de los siguientes mecanismos (Fig. 1): al 26.7\% les creció la raíz secundaria embrional y les rebrotó la raíz principal justo arriba del corte, mientras que a un $20 \%$ solo les rebrotó la principal, y a un 13.3\% les creció solo la secundaria embrional. Por otra parte, no se observó el desarrollo de raíz alguna al resto de las plántulas sobrevivientes por lo que se presume se mantuvieron vivas debido a las reservas de la semilla.

Las diferencias en la longitud de la raíz primaria o principal fueron significativas entre los tratamientos solo para las primeras dos mediciones $\left(\mathrm{F}_{6}=18.6, \mathrm{~F}_{7}=16.0, \mathrm{p}<0.01\right)$. Pero para el tercer periodo no lo fueron gracias a la capacidad de rebrote y crecimiento de la nueva raíz principal en las plántulas con el tratamiento "srp" $(\mathrm{H}=0.67, \mathrm{p}=0.72)$. El rebrote de esta raíz alcanzó un 70\% de la longitud obtenida en las plantas control al final de la investigación.

La raíz secundaria, en general, mostró un desarrollo más lento al compararla con la raíz primaria o principal (Fig. 3). Sin embargo, cuando esta última fue seccionada, se notó un crecimiento vigoroso de la raíz secundaria embrional en algunas plántulas, especialmente hacia el final del experimento (ocho meses de germinada, cinco meses después de cortada). Éstas inclusive alcanzaron longitudes similares a los rebrotes de la raíz principal (efecto compensatorio), presumiblemente facilitado por las reservas presentes en la semilla. Comparado con las de las plantas control su crecimiento fue mayor, y llegaron a alcanzar hasta un $60 \%$ de la longitud de la raíz primaria en este grupo ct. No se detectaron diferencias estadísticas en ninguno de los casos $(\mathrm{H} \leq 5.4, \mathrm{p} \geq 0.068)$.

La biomasa de las partes vegetativas también sufrió variaciones según los tratamientos (Fig. 5). Se encontraron diferencias significativas a los seis y siete meses para la biomasa del vástago $\left(\mathrm{H}_{\mathrm{ba} 6}=7.388, \mathrm{p}_{\mathrm{ba6}}=0.025 ; \mathrm{F}_{\mathrm{ba} 7}=7.205\right.$, $\left.\mathrm{p}_{\mathrm{ba}}=0.003\right)$, siendo las plántulas del control las que más acumularon. 

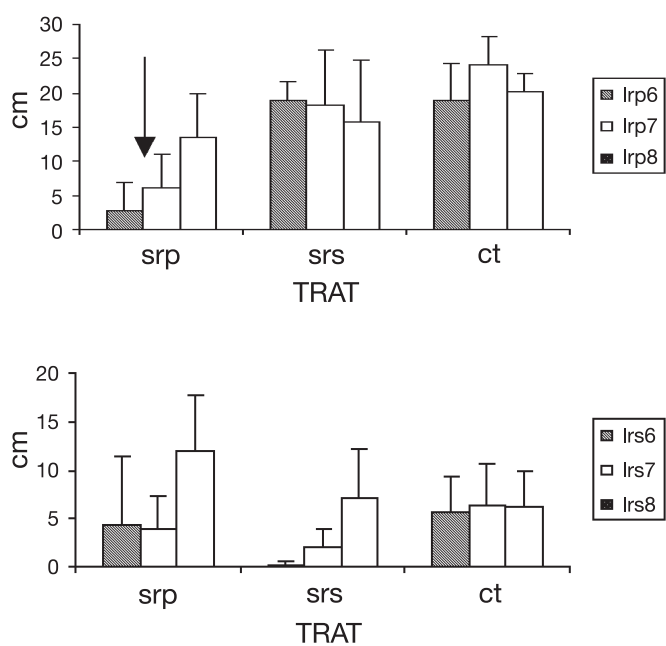

Fig. 3. Longitud de la raíz primaria o principal (lrp) y raíz secundaria embrional (lrs) promedio (+ desviación estándar) de Garcinia intermedia en tres periodos de muestreo (6, 7 y 8 meses de germinado) según los tratamientos (TRAT: srp= sin raíz primaria; srs= sin raíz secundaria; $\mathrm{ct}=$ control). La flecha indica momento del rebrote de la raíz principal.

Fig. 3. Average (+ standard deviation) length of the primary (lrp) and secondary embrionic (lrs) roots of Garcinia intermedia on three sampling periods (6, 7 and 8 months after germination) according to treatments (TRAT: $\operatorname{srp}=$ without the primary root; srs= without the secondary root; $\mathrm{ct}=\mathrm{con}-$ trol). Arrow indicates when the primary root sprouted.

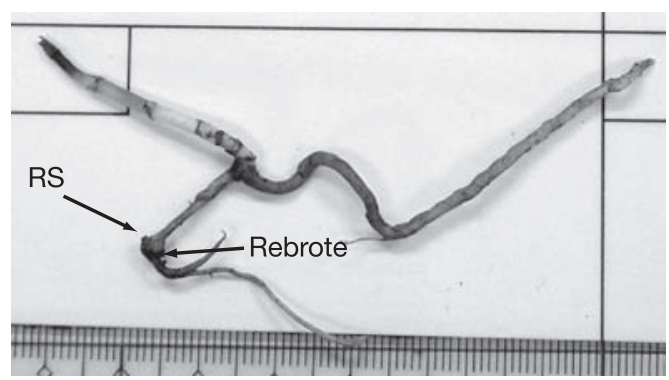

Fig. 4. Plántula de Garcinia intermedia recién germinada donde se muestra el rebrote de la raíz secundaria embrional luego que ésta fue cortada.

Fig. 4. Recently germinated Garcinia intermedia seedling with the sprout of the embrionic secundary root, after it was cut off.
En cuanto a la biomasa radical, en los dos primeros muestreos se encontraron diferencias significativas entre los tratamientos $(\mathrm{H}$ brpr6 $=$ 15.89, $\mathrm{p}_{\text {brpr6 }}<0.001 ; \mathrm{F}_{\text {brpr7 }}=5.625$, $\left.\mathrm{p}_{\text {brpr }}=0.011\right)$, siendo las plántulas del control las que nuevamente presentaron el mayor peso seco. Tal como se mencionó, a varias de las plántulas del tratamiento "srp" les rebrotó una raíz principal arriba del corte de la primaria, y su peso fue aumentando a través del tiempo (Fig. 5).

La raíz secundaria mostró pesos muy bajos comparados con la primaria o principal (14 veces menos en promedio, Fig. 5). Éstas tendieron a desaparecer o permanecieron muy pequeñas en las plantas control. Sin embargo, se observa que algunas de las plántulas a las que se les cortó la raíz primaria (a un $13.3 \%$ ), se estimuló el crecimiento de la raíz secundaria embrional, tal como se acotó anteriormente (Fig. 6). Se encontraron diferencias significativas en los dos primeros muestreos $\left(\mathrm{H}_{\text {brs6 }}=15.002, \mathrm{p}_{\text {brs6 }}=0.001 ; \mathrm{H}_{\text {brs } 7}=16.809\right.$, $\left.\mathrm{p}_{\text {brs7 }}<0.001\right)$. Sin embargo, no mostró una tendencia a incrementar con el tiempo.

La biomasa de la semilla fue menor en las plántulas del control, especialmente cuando se comparan con las del "srp" (Fig. 4). La prueba de Kruskal-Wallis mostró diferencias marginalmente significativas para las primeras dos fechas de muestreo $\left(\mathrm{H}_{\mathrm{bsem} 6}=5.857, \mathrm{p}_{\mathrm{bsem} 6}=\right.$ 0.053; $\mathrm{H}_{\text {bsem7 }}=5.471, \mathrm{p}_{\text {bsem7 }}=0.065$ ).

En la figura 7 se muestra el porcentaje de humedad promedio de las semillas (sin cubierta seminal) y las plántulas según los tratamientos. En general, los valores más bajos se observaron en las plántulas sin la raíz primaria, especialmente en la primera fecha de extracción por el desequilibrio entre la biomasa aérea y radical. Sin embargo, en las siguientes fechas el contenido de humedad de éstas fue aumentando hasta alcanzar valores intermedios como los de las plántulas del tratamiento "srs", y en algunos casos, semejantes a los del control. En el Cuadro 1 se observan 

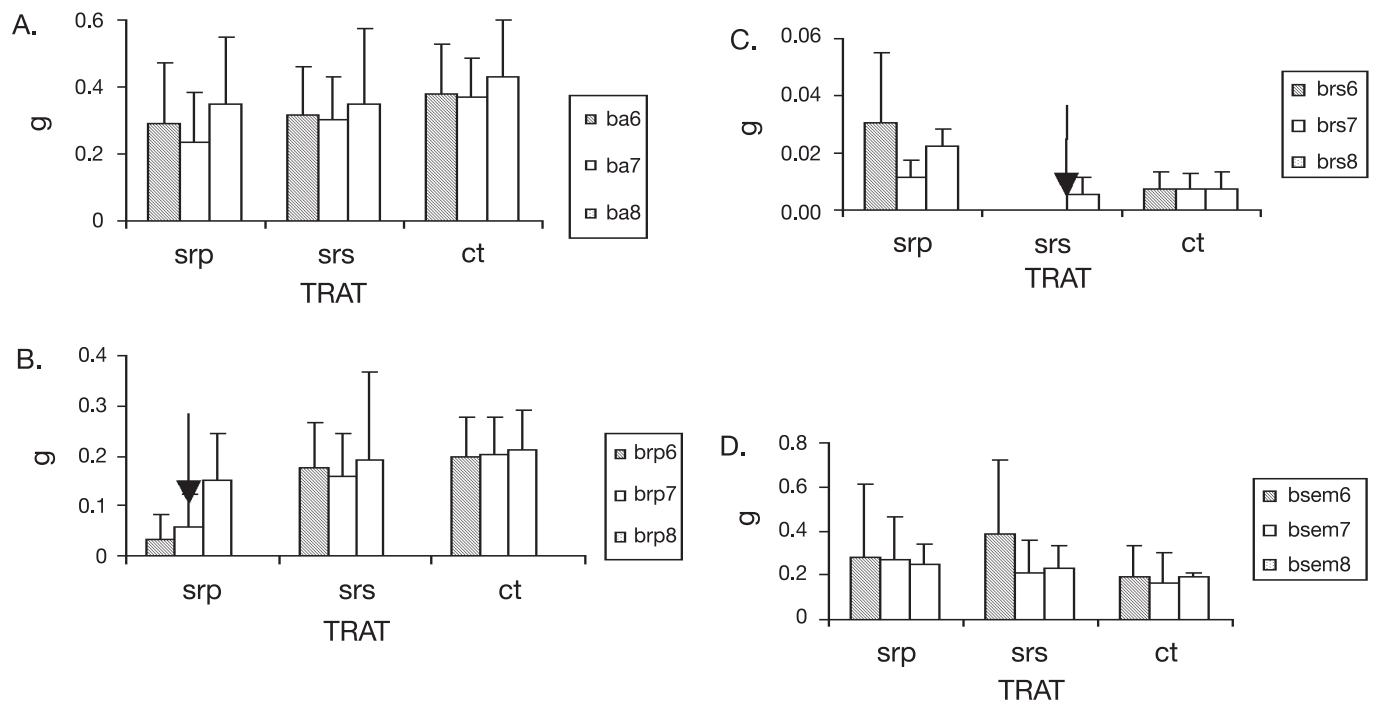

Fig. 5. Biomasa (peso seco) promedio (+ desviación estándar) del vástago (ba, A.), la raíz primaria o principal (brp, B.), la raíz secundaria (brs, C.) y la semilla (bsem, D.) en Garcinia intermedia en tres periodos de muestreo (6, 7 y 8 meses de germinado) según los tratamientos (TRAT: srp= sin raíz primaria; srs= sin raíz secundaria; ct= control). La flecha indica rebrote de raíz principal (B.) y a veces de la secundaria embrional (C.).

Fig. 5. Average (+ standard deviation) aerial (ba, A), primary root (brp, B.), secondary root (brs, C.), and seed (bsem, D.) biomass (dry weight) of Garcinia intermedia on three sampling periods (6, 7 and 8 months after germination) according to treatments (TRAT: $\mathrm{srp}=$ without the primary root; srs= without the secondary root; $\mathrm{ct}=\mathrm{control}$ ). The arrow shows when the primary root sprouted (B.), and in some cases the secondary embryonic root (C.).

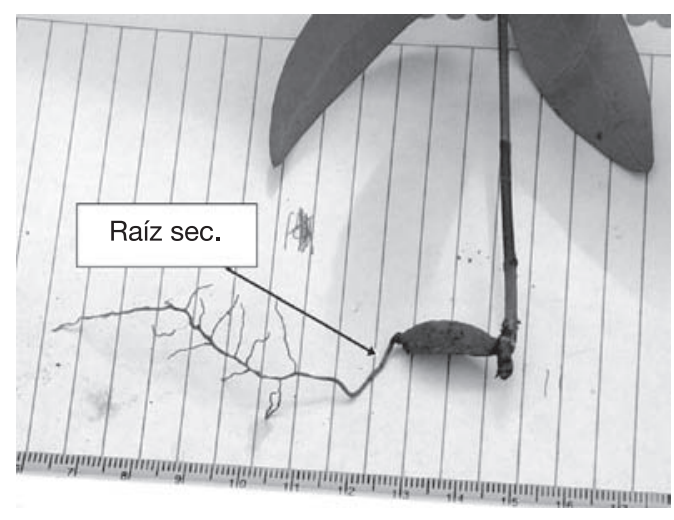

Fig. 6. Plántula de Garcinia intermedia con seis meses de germinada donde se muestra el crecimiento de la raíz secundaria, luego de cortada la raíz primaria.

Fig. 6. Garcinia intermedia seedling six months after germination with the secondary root growth after the primary root was cut off. las fechas en las que se alcanzaron diferencias estadísticamente significativas.

\section{DISCUSIÓN}

En general, las plántulas control fueron las que tuvieron un mejor crecimiento del vástago y de la raíz primaria. Además, presentaron un mayor contenido de humedad en su biomasa y un menor peso seco de la semilla todavía adherida a la plántula. Le siguieron aquéllas a las que se les cortó la raíz secundaria embrional. Al parecer, esta raíz tuvo un efecto en el crecimiento de las plántulas, aunque su eliminación aparentemente no produjo diferencias significativas bajo las condiciones del invernadero. Observaciones de plántulas 

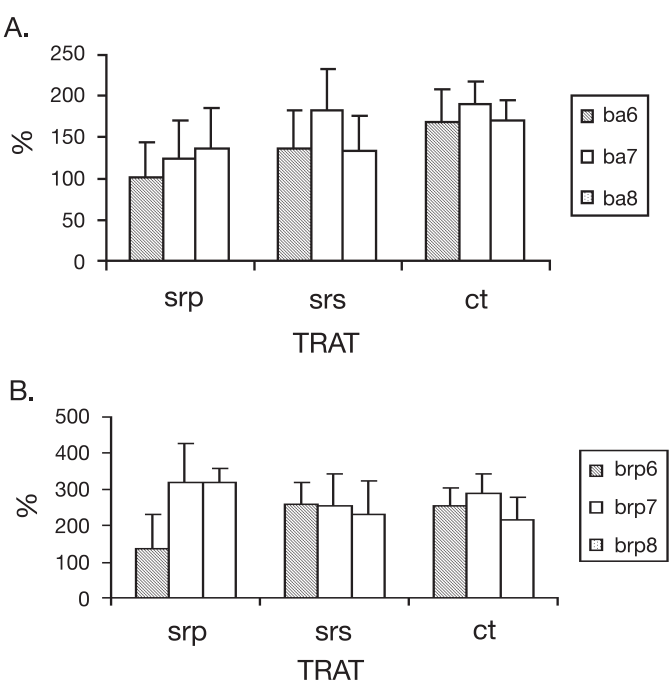

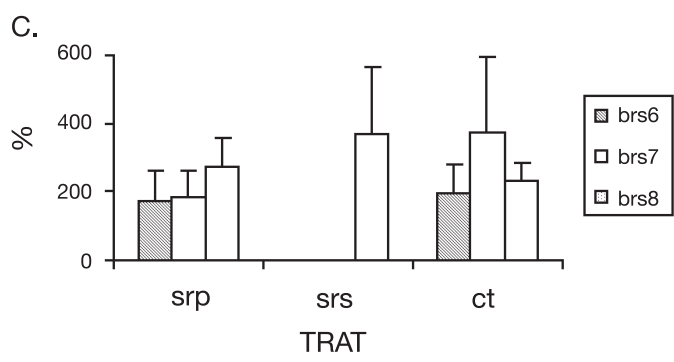

D.

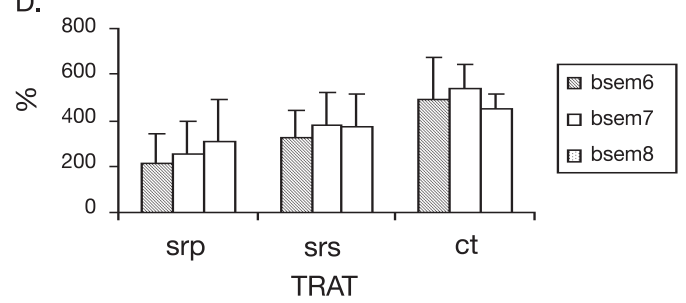

Fig. 7. Porcentaje de humedad promedio (g de agua/g peso seco,+ desviación estándar) en la biomasa del vástago (ba, A.), la raíz primaria o principal (brp, B.), la secundaria (brs, C.), y la semilla (bsem, D.) en Garcinia intermedia en tres periodos de muestreo (6, 7 y 8 meses de germinado) según los tratamientos (TRAT: srp= sin raíz primaria; srs= sin raíz secundaria; $\mathrm{ct}=\mathrm{control})$.

Fig. 7. Average (+ standart deviation) humidity percentage (g of water/g dry weight) of the aerial (ba, A), primary root (brp, B), secondary root (brs, C.), and seed (bsem, D.) biomass (dry weight) of Garcinia intermedia on three sampling periods (6, 7, and 8 months after germination) according to treatments (TRAT: $\operatorname{srp}=$ without the primary root; srsn= without the secondary root; $\mathrm{ct}=$ control).

extraídas en el campo mostraron que esta raíz secundaria desaparece temprano (W. Marín y J. Di Stefano, obs. pers.), aunque en pocas ocasiones (menos de un 1\% de las plántulas extraídas del bosque) se han observado con dos raíces bien desarrolladas, una de las cuales se cree corresponde a un crecimiento de la raíz secundaria embrional.

Las plántulas más afectadas fueron a las que se les cercenó la raíz primaria. Sin embargo, la mayoría (+90\%) logró sobrevivir, y muchas de ellas inclusive recuperar una condición similar a la de los otros tratamientos en términos de su crecimiento y biomasa. Esto posiblemente se debió a las reservas de la semilla, a las favorables condiciones del experimento y a la capacidad de esta especie de regenerar su raíz principal y/o fortalecer la raíz secundaria embrional (por lo menos en un $60 \%$ de ellas). Este aspecto resultó muy interesante ya que normalmente si la raíz primaria es destruida, las plántulas mueren.
Otro factor que favoreció la supervivencia de estas plántulas, fue probablemente el látex, el cual es muy abundante en esta especie. Este látex es pegajoso, espeso, seca rápidamente y es probable que tenga efectos bactericidas o funguicidas. En extractos de otras especies de este género se han encontrado principios químicos activos eficaces en el control de parásitos, virus y contra enfermedades en seres humanos (Permana et al. 2001). Por otra parte, la menor longitud de la raíz primaria hacia el final de la investigación en las plántulas del control y sin la raíz secundaria, se pudo deber al tamaño de las bolsas que limitó su crecimiento.

En varias plántulas también se les observó un aumento de la longitud de la raíz secundaria en el tratamiento en la que ésta fue removida, la cual se debió a dos factores: a un rebrote de la misma (Fig. 4) o a un crecimiento de ésta raíz enrollada dentro de la cubierta seminal en algunas de ellas (Fig. 3). 
CUADRO 1

Tratamientos que alcanzaron niveles estadísticamente significativos para el contenido de humedad de la biomasa

TABLE 1

Statistically significant treatments for biomass moisture content

$\begin{array}{ccc}\text { Tratamiento-fecha } & \begin{array}{c}\text { Valor } \\ \text { observado }\end{array} & \begin{array}{r}\text { Probabilidad } \\ \text { alcanzada }\end{array} \\ \text { ba\%6* } & \mathrm{F}^{* *}=6.021 & 0.007 \\ \text { ba\%7 } & \mathrm{H}^{* *}=8.960 & 0.011 \\ \text { brp\%6 } & \mathrm{H}=8.960 & 0.011 \\ \text { brp\%8 } & \mathrm{F}=3.451 & 0.051 \\ \text { brs\%6 } & \mathrm{H}=12.451 & 0.002 \\ \text { brs\%7 } & \mathrm{H}=11.884 & 0.003 \\ \text { bsem\%6 } & \mathrm{F}=8.950 & 0.001 \\ \text { bsem\%7 } & \mathrm{F}=10.512 & <0.001 \\ \text { bsem\%8 } & \mathrm{H}=6.976 & 0.031\end{array}$

*ba= vástago (shoot); brp= raíz primaria o principal (primary root); brs= raíz secundaria (secondary root); sem= semilla (seed) a los 6, 7 u 8 meses después de la germinación (6, 7 or 8 months alter germination).

**F y $\mathrm{H}=$ índices observados de Fisher o Kruskal-Wallis (Fisher o Kruskal-Wallis observed indixes).

La raíz secundaria embrional se ha observado en otras cluseáceas (E. Flores, com. pers.), pero ésta es la primera vez que se informa de ella y se elabora un experimento para determinar su potencial para la supervivencia y crecimiento de las plántulas de Garcinia cuando la raíz primaria es dañada. Con base en este estudio, se concluye que la raíz secundaria embrional logró tomar fuerza en al menos el 40\% de las plántulas cuya raíz primaria fue dañada, por lo cual se considera que ésta pudo jugar un papel importante en la recuperación de las plántulas afectadas. Sin embargo, esta especie también mostró una alta capacidad para regenerar su sistema radical a través del rebrote de la raíz principal. Se deben hacer más investigaciones para determinar la posible interacción de esta raíz secundaria con las reservas de la semilla y bajo condiciones más estresantes.

\section{AGRADECIMIENTOS}

A la Vicerrectoría de Investigación de la Universidad de Costa Rica por el financiamiento parcial del proyecto No. 111-A3-029. A los personeros de la Universidad de la Paz por permitirnos el ingreso a la Zona Protectora "El Rodeo".

\section{RESUMEN}

En pruebas preliminares de germinación con semillas de Garcinia intermedia (Clusiaceae) se observó que ésta producía dos tipos de raíces: la primaria y una secundaria embrional que atraviesa longitudinalmente la semilla. Para determinar su posible papel en la supervivencia y crecimiento de esta especie, se analizaron 90 plántulas con al menos seis meses de germinadas en bolsas plásticas con suelo orgánico ubicadas en un invernadero, a las que se les aplicó uno de los siguientes tratamientos: corte de la raíz primaria o la secundaria embrional, además de un grupo control con ambas raíces (30 repeticiones de cada uno). Luego de tres meses se empezaron a extraer 10 plántulas/ mes/tratamiento a los que se les midió la altura total, diámetro basal, longitud de la raíz primaria y secundaria, y la biomasa del vástago, raíz y semilla sin la cubierta seminal. Las plántulas que obtuvieron el mayor crecimiento fueron las del control, seguidas por aquéllas sin la raíz secundaria. Sin embargo, más del $90 \%$ de las plántulas a las que se les cortó la raíz primaria, sobrevivieron después de cinco meses de haberse aplicado el tratamiento, en parte debido a una muy alta capacidad de recuperación de su sistema radical, ya sea produciendo rebrotes de la principal (20\%), aumentando el crecimiento de la raíz secundaria (13.3\%), o ambos (26.7\%), y a las reservas de la semilla. La longitud de los rebrotes de la raíz principal fue estadísticamente significativa en las primeras dos extracciones mensuales de plantas, comparado con las del control $\left(\mathrm{F}_{6}=18.6, \mathrm{~F}_{7}=16.0\right.$, $\mathrm{p}<0.01$ ), pero no en la tercera medición. Bajo las condiciones de invernadero, la biomasa total de las plántulas cuya raíz primaria fue cortada, alcanzó valores semejantes a la de los otros tratamientos cinco meses después de haberse aplicado el tratamiento.

Palabras clave: raíz primaria, raíz secundaria, germinación hipogenia, bosque premontano húmedo tropical, Costa Rica.

\section{REFERENCIAS}

Augspurger, C.K. 1984. Seedling survival of tropical tree species: Interactions of dispersal distance, light gaps, and pathogens. Ecol. 65: 1705-1712. 
Cascante, A. \& A. Estrada. 2001. Composición florística y estructura de un bosque húmedo premontano en el Valle Central de Costa Rica. Rev. Biol. Trop. 49: 213-225.

Clark, D.B. \& D.A. Clark. 1989. The role of physical damage in the seedling mortality regime of a neotropical rain forest. Oikos 55: 225-230.

Dirzo, R. 1987. Estudios sobre las interacciones planta-herbívoro en "Los Tuxtlas”, Veracruz. Rev. Biol. Trop. 35 (Supl.1): 119-132.

Farji-Brener, A.G., S. Durán, A. Valerio, E. Herbas, M. Castañeda, J. Ochoa \& M. Romo. 2005. La semilla de Campsiandra angustifolia (Fabaceae: Caesalpiniodeae) como un reflejo de las presiones selectivas sobre su dispersión y establecimiento. Rev. Biol. Trop. 53 (1-2): 63-71.
Fournier, L.A. \& S. Salas. 1967. Tabla de vida para el primer año de la población de Dipterodendron costaricense Ralk. Turrialba 17: 348-350.

García-Robledo, C. 2005. Comparación de dos métodos para medir herbivoría. ¿Es la herbivoría en el Neotrópico mayor de lo que creemos? Rev. Biol. Trop. 53 (1-2): 111-114.

Permana, D., N. Hj. Lajis, M.M. Mackeen, A.M. Ali, N. Aimi, M. Kitajima \& H. Takayama. 2001. Isolation and Bioactivities of Constituents of the Roots of Garcinia atroviridis. J. Natur. Prod. 64: 976-979.

Zamora, N., Q. Jiménez \& L. J. Poveda. 2004. Árboles de Costa Rica. Vol. III. INBIO, Heredia, Costa Rica. 556 p. 